\title{
APPLICATION AND DYNAMIC SIMULATION OF IMPROVED GENETIC ALGORITHM IN PRODUCTION WORKSHOP SCHEDULING
}

\author{
Jiang, P. ${ }^{* * * * \# ; ~ D i n g, ~ J . ~ L . ~}{ }^{*} \&$ Guo, Y.* \\ *School of Information Technology, Jiangxi University of Finance and Economics, Nanchang 330013, \\ China \\ ${ }^{* *}$ Yiwu Industrial and Commercial College, Yiwu 322000, China \\ E-Mail: ywiccjp@ywu.cn (\# Corresponding author)
}

\begin{abstract}
From the point of view of combining theories with practice, in order to better realize the effective management of production workshop scheduling and thus improve the competitiveness of manufacturing enterprises in the market, this study focuses on the simulation of production workshop scheduling based on genetic algorithm. Firstly, the study analyses the concept and characteristics of production workshop scheduling, and then proposes the objective function and scheduling rules of production workshop scheduling; the study also introduces the genetic algorithm to combine the scheduling rules, which are regarded as the genetic gene to realize the optimization of the production scheduling through the simulation calculation; in addition, in order to satisfy the dynamic production environment of manufacturing enterprises, the study put forward a dynamic scheduling model; finally, the dynamic simulation of the production workshop scheduling system is realized through the mixed programming of Matlab and VC. The simulation results show that the dynamic model can reduce the scheduling times and improve the stability of dynamic scheduling, and has a positive guiding significance for the production workshop scheduling of manufacturing enterprises.

(Received, processed and accepted by the Chinese Representative Office.)
\end{abstract}

Key Words: Production Workshop Scheduling, Genetic Algorithm, Dynamic Model, Dynamic Simulation

\section{INTRODUCTION}

The process of global integration leads to the increasingly fierce competition in the enterprise market. The production cycle and quality of the products are the key factors of manufacturing enterprises to meet the diversified demands of the customers. How to improve the production efficiency and reduce the cost under the changeable market environment is the key for manufacturing enterprises to adapt to the market environment. Manufacture Resource Planning (MRP) and Enterprise Resource Planning (EPR) are the macro management methods for modern manufacturing enterprises to achieve lot size, set delivery time, implement workshop constraints and conduct resource allocation [1,2]. However, the most basic unit that affects the efficiency and quality of enterprise production is still the workshop of the enterprise. Only the realization of the agility of the workshop production and improvement of the management level and production efficiency is the key to raise the competitiveness of the enterprise.

At present, with the introduction of foreign advanced management technology and practice accumulation, the workshop management control work in China has completed workshop operation planning, process scheduling, operation management, inventory control and other software, and achieved certain effects [3]. In the simulation research of workshop scheduling in China, there are rule-based method, intelligent scheduling method, domain search method, simulated annealing method and so on. However, there are the following problems in production management: it's hard to accurately and timely grasp the actual 
production conditions in the plan process, it's impossible to obtain the practical operation plan during the production process to guide the management personnel the production workshop, and operators cannot follow the state data of the products during the production process, and effectively control the in-process products and inventory, while the users can have no idea about the execution of the order before the delivery [4]. In simulation research, some problems such as imperfect modelling method and unclear objective function of production scheduling affect the accuracy and adaptability of simulation model.

In order to realize the dynamic simulation of production workshop scheduling in enterprises, based on the analysis of relevant concepts, the study establishes a mathematical model conforming to the production scheduling rules of the enterprise, and proposes an optimized solution based on genetic algorithm; at the same time, the dynamic simulation analysis of relevant parameters of production workshop scheduling is carried out, and the dynamic simulation and man-computer interaction for production workshop scheduling are completed under the mixed programming of Matlab and VC [5]. The study realizes the application of genetic algorithm in the dynamic scheduling of workshops. The simulation results and system implementation are of great significance in guiding the scientific scheduling management of workshops.

\section{OVERVIEW OF PRODUCTION SCHEDULING AND GENETIC ALGORITHM}

\subsection{Production scheduling}

\section{(1): Definition of production scheduling}

The so-called production scheduling is the reasonable arrangement and plan made by the enterprise in the process of production management. In particular, the production scheduling of the enterprise can be described as the right allocation management in a production process, in which there are $N$ machines in a workshop for production and processing, $M$ processes are required for a product, each process is distributed on different machines, each of which can only process specific workpieces, and the next process can be performed only after one process is completed [6].

(2): Classification of production workshop scheduling

According to the characteristics of workpiece processing, the scheduling can be divided into static scheduling and dynamic scheduling: in static scheduling, the parts are ready before starting, without considering the machine damage, new workpiece demand, advance delivery and other factors; in dynamic scheduling, considering various sudden conditions in the workpiece process, the scheduling is required to respond to the change of the workshop, and the re-scheduling can be performed to ensure the optimization of the processing performance in the event of an emergency [7].

(3): Basic idea of production workshop scheduling system

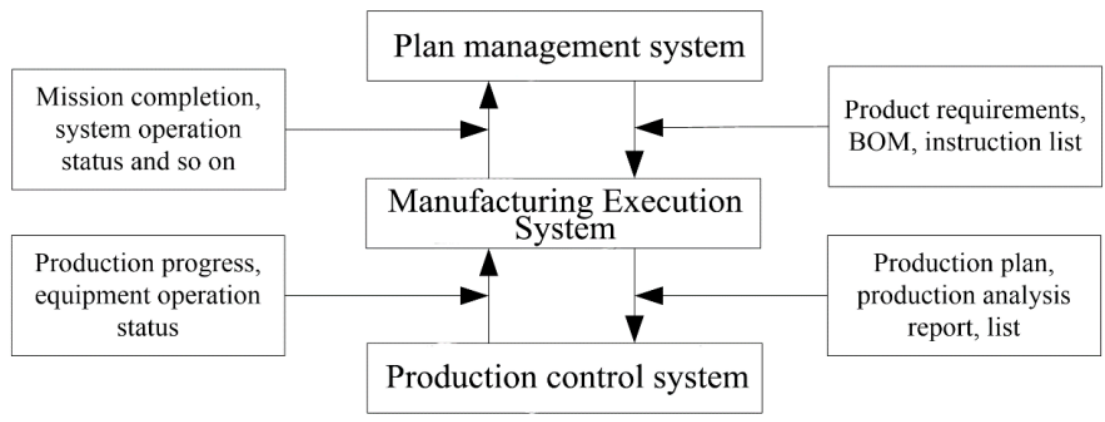

Figure 1: Information flow among Plan layer/MES/Control layer. 
In order to make the scheduling result meet the actual production demand as much as possible, the workshop production should be based on a certain scheduling idea. Fig. 1 shows the information flow among Manufacturing Execution System (MES) and planning layer and control layer, reflecting the basic idea of production workshop scheduling [8].

(4): Mathematical definition of production workshop scheduling

The optimization goal of production workshop scheduling is to make the production time of workpieces shortest, without exceeding the due time. The scheduling of production workshop can be described as follows [9]:

a) Define $P$ as a set of $n$ workpieces to be processed, and its expression is shown in Eq. (1):

$$
P=\left\{P_{i}\right\} ; i=1,2, \ldots, n
$$

b) Assuming $P_{i}$ has $r_{j}$ processes, define $O_{i j}$ is its $j^{\text {th }}$ process, and the corresponding working time quota is $t_{i j}$, as shown in Eq. (2):

$$
P_{i}=\left\{O_{i j}\right\} ; j=1,2, \ldots, r_{i}
$$

Working time quota of the process:

$$
t_{i}=\left\{t_{i j}\right\} ; j=1,2, \ldots, r_{i}
$$

c) Define $M$ as a set of $m$ devices, then,

$$
M=\left\{M_{k}\right\} ; k=1,2, \ldots, m
$$

d) Due to the difference in processing ability, different machines have different processing time for the same process. $t_{i j}\left(j=1,2, \ldots, r_{i}\right)$ in $M$ matrices indicates working time quota of the process for different workpieces in different machines.

$T_{M 1}=\left[\begin{array}{cccc}t_{11} & t_{12} & \ldots & t_{1 L} \\ t_{21} & t_{22} & \ldots & t_{2 L} \\ \ldots & \ldots & \ldots & \ldots \\ t_{n 1} & t_{n 2} & \ldots & t_{n L}\end{array}\right], T_{M 2}=\left[\begin{array}{cccc}t_{11} & t_{12} & \ldots & t_{1 L} \\ t_{21} & t_{22} & \ldots & t_{2 L} \\ \ldots & \ldots & \ldots & \ldots \\ t_{n 1} & t_{n 2} & \ldots & t_{n L}\end{array}\right], \ldots, T_{M m}=\left[\begin{array}{cccc}t_{11} & t_{12} & \ldots & t_{1 L} \\ t_{21} & t_{22} & \ldots & t_{2 L} \\ \ldots & \ldots & \ldots & \ldots \\ t_{n 1} & t_{n 2} & \ldots & t_{n L}\end{array}\right]$

e) Define $s t_{i} ; s t_{i}(i=1,2, \ldots, n)$ as delivery time of each workpiece.

f) The optimal scheduling objective function is as follows:

$$
\begin{gathered}
\text { MIN } \sum_{i=1}^{n} f\left(x_{i}\right) \\
f\left(x_{i}\right)=0 ; x_{i}<s t_{i} ; \\
f\left(x_{i}\right)=x_{i}-s t_{i} ; x_{i}>s t_{i} ;
\end{gathered}
$$

where, $x_{i}$ is actual completion time of each workpiece and $n$ is the number of workpieces.

(5): Scheduling rules of production workshop

In order to minimize the completion time and address parallel multi-machine scheduling problem, a genetic algorithm base on combination rule is proposed in this study. The genetic algorithm is used to optimize the combination of schedule rules, so that different scheduling rules are adopted in different scheduling stages to improve the performance of the algorithm [10]. The rules of workshop scheduling mainly include processing amount rules, remaining time rules, and rules on the ratio of processing quantity to remaining time. The following focuses on processing quantity rules.

The processing amount of the workpieces is measured by the total time of the machined workpieces. Its calculation formula is as shown in Eq. (7):

$$
A_{t_{i j}}=\sum_{k=1}^{m} t_{i j}^{k} / m
$$


Totaltime $_{i}$ is used to represent the processing amount product $t_{i}$ of the $i^{\text {th }}$ workpiece. The scheduling can be divided into three steps:

Step 1: Quantize the processing amount, and prioritize the processing according to the processing amount, with a larger processing amount having a higher priority.

Step 2: Judge the machine idle rate. If one machine is idle, workpieces of a large processing amount should be arranged for processing. If several machines are idle, the machine that can process workpieces with the shortest processing time should be arranged. If the workpieces with the highest priority cannot be processed by the idle machine, the subpriority workpieces should be selected for the idle machine.

Step 3: Re-quantify the processing amount after the completion of a process, that's, product $_{i}=$ product $_{i}-A t_{i j}$. Re-arrange the priority and repeat Step 2 until all processes are finished.

For example, there are 5 workpieces in total, 3 processes are required for each workpiece, and the time consumed by each machine for each process can be expressed as:

$$
T_{M 1}=\left[\begin{array}{lll}
4 & 7 & 0 \\
3 & 0 & 7 \\
3 & 0 & 0 \\
5 & 7 & 0 \\
0 & 0 & 4
\end{array}\right], T_{M 2}=\left[\begin{array}{lll}
0 & 7 & 5 \\
0 & 6 & 9 \\
4 & 5 & 0 \\
0 & 0 & 6 \\
0 & 9 & 0
\end{array}\right], \ldots, T_{M m}=\left[\begin{array}{ccc}
6 & 9 & 5 \\
0 & 0 & 8 \\
5 & 0 & 6 \\
7 & 9 & 5 \\
6 & 10 & 7
\end{array}\right]
$$

Table I shows the time when the workpiece is completed, where positive or zero indicates that the workpiece can be completed on time or in advance, and negative indicates that the workpiece is not completed on time.

Table I: Scheduling instance based on product.

\begin{tabular}{|c|c|c|c|}
\hline Workpiece number & Delivery time & Completion time & Workpiece completed in advance \\
\hline $\mathbf{1}$ & 29 & 33 & -4 \\
\hline $\mathbf{2}$ & 30 & 30 & 0 \\
\hline $\mathbf{3}$ & 33 & 22 & 11 \\
\hline $\mathbf{4}$ & 34 & 28 & 6 \\
\hline $\mathbf{5}$ & 36 & 23 & 13 \\
\hline
\end{tabular}

\subsection{Genetic algorithm}

(1): Basic concepts and steps of implementation

Genetic algorithm is a search optimization algorithm to simulate the evolution process of natural organisms. It can improve the adaptability of population through selection, mutation and heredity [11]. It is widely used in complex problems, production scheduling, non-linear problems and others [12]. The specific steps of the genetic algorithm are as follows:

Step 1: Randomly generate a set of initial populations;

Step 2: The fitness value of each individual is calculated as an evaluation criterion. If the given requirement is met, or the set value is reached, the searching process is ended, and the optimal individual of the population is the solution of the given problem.

Step 3: Individuals are selected according to the probability proportional to their fitness, and new individuals are formed through exchange and mutation operations.

Step 4: Turn to Step 2 [13].

(2): Application of genetic algorithm in production workshop scheduling

a) Coding and decoding

According to the example in 2.1, the chromosome length of 5 types of workpieces for 3 steps is 15 . Their chromosomes can be expressed as:

\begin{tabular}{|l|l|l|l|l|l|l|l|l|l|l|l|l|l|l|}
\hline 2 & 2 & 1 & 0 & 1 & 1 & 0 & 2 & 0 & 0 & 1 & 1 & 2 & 1 & 2 \\
\hline
\end{tabular}


Among them, 0, 1 and 2 represent three kinds of scheduling rules respectively. In workshop production scheduling, chromosome genes are selected according to sequence, and scheduling rules are selected through gene coding to arrange the production of workpieces.

b) Population initialization

Preliminary optimization and random generation are methods of population initialization. There are 100 individuals with chromosome length of 15 in the initialization population, 100 individuals are different and randomly generated.

c) Crossover operator

The chromosomes are divided into two subsets A and B, and the parent 1 genes belonging to $\mathrm{A}$ are classified into son 1 type and those belonging to B into son 2 type; the parent 2 genes belonging to $\mathrm{A}$ are classified into son 2 type and those belonging to $\mathrm{B}$ into son 1 type. The following gene combinations are obtained:

Parent 1: $\{1,1,2,0,0,2,1,2,1,2,1,2,0,1,2\}$;

Parent 2: $\quad\{0,0,2,2,1,2,0,2,1,0,2,2,0,1,1\}$

Son 1: $\quad\{0,0,2,2,1,2,1,2,1,2,1,2,0,1,2\}$;

Son 2: $\quad\{1,1,2,0,0,2,0,2,1,0,2,2,0,1,1\}$.

The final chromosome is then selected from the four individuals as the individual in the next population according to the selection strategy.

d) Mutation operator

Randomly select an individual in the population, randomly split it into two rows, and then swap the order to form a new individual.

e) Selection

Select the best individuals in the population, and eliminate inferior individuals. Wherein, the adaptation value is a reference index for selection.

f) Terminating conditions

Judge whether the algorithm converges by observing the number of iterations $N$ and the convergence curve, and adjust it in real time [14].

\section{RESEARCH ON PRODUCTION WORKSHOP SCHEDULING ALGORITHM BASED ON GENETIC ALGORITHM}

The internal and external environment of the enterprise is changing all the time, and the enterprise production is faced with many unpredictable factors. Based on the theory of the second chapter herein, a dynamic workshop scheduling method is proposed in this study, and the reliability of the algorithm is analysed with examples.

\subsection{Dynamic scheduling calculation model}

The dynamic change will disturb the production plan of workpieces, even affect the production of the whole workshop, and the dynamic adjustment in the face of the change is the basic requirement of the workshop [15].

The dynamic scheduling of production workshop is to implement a re-scheduling when a kind of workpieces are finished or finished in advance or delayed, the urgent workpiece are inserted, and the machine is damaged or repaired, so that the scheduling events occur frequently. If there is an impact, restart a scheduling. When the rescheduling event occurs again, the above process is repeated until all the workpieces are finished [16]. The mathematical model of the dynamic change of the workshop is shown in Eq. (9):

$$
\text { fitness }=\min \left(\sum_{i=1}^{n} f\left(x_{i}\right)\right)
$$




$$
\begin{gathered}
f\left(x_{i}\right)=0, x_{i}<T_{i} \\
f\left(x_{i}\right)=x_{i}-T_{i}, x_{i}>T_{i}
\end{gathered}
$$

$i$ refers to various workpieces $(i=1,2, \ldots, n) ; j$ refers to working procedures of various workpieces; $I$ is the first working procedure of rescheduling $i$-type workpieces for processing; $k$ refers to process on the $k^{\text {th }}$ machine; $x_{i}$ represents the actual completion time of the workpiece; $T_{i}$ is the given delivery time of the workpiece; $f\left(x_{i}\right)$ indicates the delay time of each type of workpieces; fitness is a function of fitness; $t_{e}$ is the start time of rescheduling; $t_{i j}^{k}$ is the time required by the $k^{\text {th }}$ machine for the $j^{\text {th }}$ process, $c_{i j}$ is difference between the start time of the $j^{\text {th }}$ process of processing the $i$-type workpieces from $t_{e}$ and the completion time of the $j-1$ process of the $i$-type workpieces. If the $j-1$ process is completed before $t_{e}, c_{i j}$ is the difference between the start time of the $j^{\text {th }}$ process of processing the $i$-type workpieces and $t_{e}$ [17].

\subsection{Research on simulation of dynamic scheduling model}

Different from static simulation, dynamic simulation can adapt to the workshop workpiece processing environment and respond quickly when the equipment is damaged, the expiration time of the workpiece is changed, etc. [18, 19].

(1): Advance or delay of the process

For advance or delay of the process, the dynamic scheduling model of workshop production is simulated as follows:

Step 1: Carry out production according to the plan.

Step 2: After each process is finished, judge whether it is produced according to the calculation. If it deviates from the plan, the chromosome is regenerated, and the chromosome length is the sum of the remaining workpiece processes.

Step 3: Return to Step 1 until all the workpieces are processed.

According to the production plan initially formulated, arrange the workpiece 1 to be processed by the machine 1; arrange the workpiece 3 to be processed by the machine 2; arrange the workpiece 5 to be processed by the machine 3 . According to the production plan, the completion time of the workpiece 1 is 4 o'clock, that of the workpiece 3 is also 4 o'clock, and that of the first process of the workpiece 5 is 7 o'clock. If the completion time of the workpiece 1 is delayed to 6 o'clock, the workpiece 3 to 5 o'clock, and the workpiece 5 to 6 o'clock under the influence of external factors such as sudden power failure or worker's proficiency degree.

The chromosome of genetic algorithm generated by static scheduling is $\{1,2,0,1,1,1,1$, $2,1,1,0,0,0,1,1\}$. The fourth gene of chromosome is 1 , which represents that the production is scheduled according to the rules. The fourth scheduling selects workpiece 3 to be processed by the machine 2 . The first 4 schedulings are as below:

$$
\left\{\begin{array}{c}
\text { part }=\{1,5,3,3\} \\
\text { plan }- \text { machine }=\{1,3,2,2\}
\end{array}\right.
$$

Planned scheduling:

$$
\left\{\begin{array}{c}
\text { plan }- \text { part }=\{1,5,3,2,1,4,2,5,4,3,2,1,3,4,5\} \\
\text { plan }- \text { machine }=\{1,3,2,1,2,3,2,3,1,2,1,2,3,2,1\}
\end{array}\right.
$$

From the fourth bit of part and machine and the fourth bit of the planned scheduling, it can be seen that the actual production and schedule have changed and the production plan needs to be reworked. The re-scheduled chromosome, machine scheduling and workpiece scheduling plans are as follows: 
chrom $=\{3,3,1,3,2,1,3,3,3,2,3,3\} ;$ plan - machine $=\{2,1,3,1,2,3,1,2,3,1,2,3\} ;$ plan - part $=\{3,2,4,1,2,5,4,2,3,5,1,4\}$.

Thus the completion times of workpieces are shown in Table II.

Table II: Dynamic scheduling instance aim at delay.

\begin{tabular}{|c|c|c|c|}
\hline Workpiece number & Delivery time & Complete time & Workpiece completed in advance \\
\hline $\mathbf{1}$ & 29 & 30 & -1 \\
\hline $\mathbf{2}$ & 30 & 25 & 5 \\
\hline $\mathbf{3}$ & 33 & 29 & 4 \\
\hline $\mathbf{4}$ & 34 & 28 & 6 \\
\hline $\mathbf{5}$ & 35 & 35 & 0 \\
\hline
\end{tabular}

(2): Machine failure

Machine failure is an unavoidable change in workshop production scheduling. The specific scheduling steps in case of machine failure in the workshop are as follows:

Step 1: Arrange production according to the plan.

Step 2: After each process is finished, send a completion signal to the scheduling system to judge whether the machine is faulty or not. In case of the fault, delete the machine from the candidate machines.

Step 3: After each process is finished, judge whether the production is carried out according to calculation, if it deviates from the plan, chromosomes are re-generated, and the chromosome length is the sum of the remaining workpiece processes.

Step 4: Return to Step 1 until all the workpieces are processed.

Consistent with initial conditions of the process advance or delay simulation, the machine 1 has a machine fault at 7 o'clock and is repaired at 17 o'clock.

Table III: Dynamic scheduling instance aim at machine malfunction.

\begin{tabular}{|c|c|c|c|}
\hline Workpiece number & Delivery time & Complete time & Workpiece completed in advance \\
\hline $\mathbf{1}$ & 29 & 22 & 7 \\
\hline $\mathbf{2}$ & 30 & 24 & 6 \\
\hline $\mathbf{3}$ & 33 & 33 & 0 \\
\hline $\mathbf{4}$ & 34 & 33 & 1 \\
\hline $\mathbf{5}$ & 35 & 39 & -4 \\
\hline
\end{tabular}

By applying genetic algorithm to dynamic scheduling of workshop production, making production plan in time can improve the ability of workshop to deal with emergencies. The simulation results can effectively track the process environment change of the workshop.

\section{SIMULATION OF PRODUCTION WORKSHOP SCHEDULING SYSTEM}

For the realization of scheduling system simulation, Matlab is used as the iterative solution tool of genetic algorithm, and Visual $\mathrm{C}++$ is used as the interface programming tool of manmachine interaction.

\subsection{Functional structure of the system}

Fig. 2 shows the main functional structure of the workshop production scheduling system. 


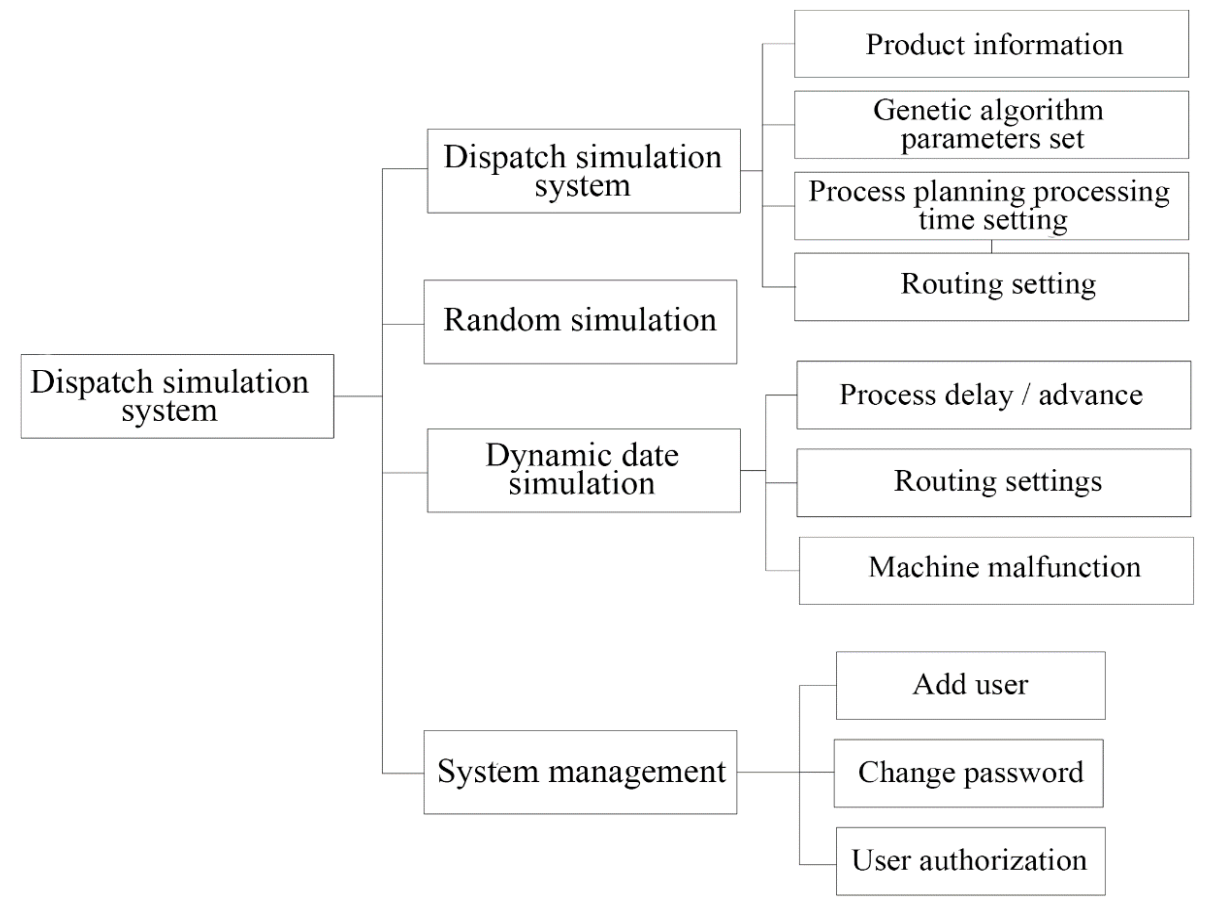

Figure 2: Function configuration of the system.

The composition of workshop scheduling simulation system includes four parts: basic data entry, random simulation, dynamic data acquisition and system management, which can satisfy many functions such as algorithm parameter setting, dynamic simulation, and user setting.

\subsection{Introduction of function module interface of the system}

Fig. 3 shows a screenshot of basic data entry module of the system. By setting the initial conditions, the system will calculate the simulation results according to the algorithm rules to guide the workshop production.

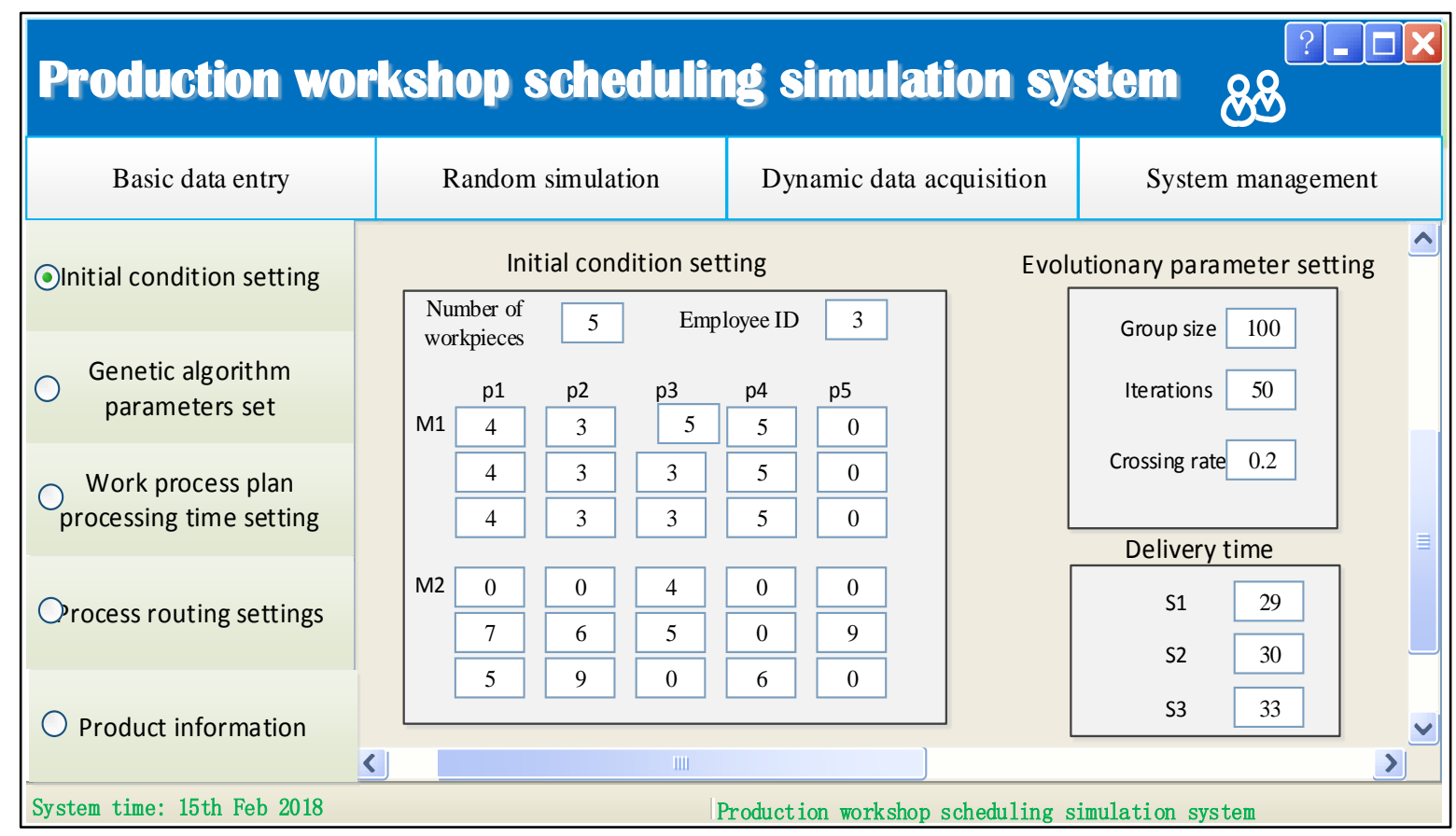

Figure 3: System screenshot. 


\subsection{Dynamic workshop scheduling simulation}

In terms of workpiece delay and machine failure in the production process, the simulation analysis is carried out on the workshop scheduling simulation platform based on genetic algorithm in the system. Set the population size of the genetic algorithm as $M=1000$, the number of iterations as 50 , the mutation rate as $p m=0.02$, and the crossover rate as $p o=0.2$. The process of the algorithm is shown in Fig. 4.

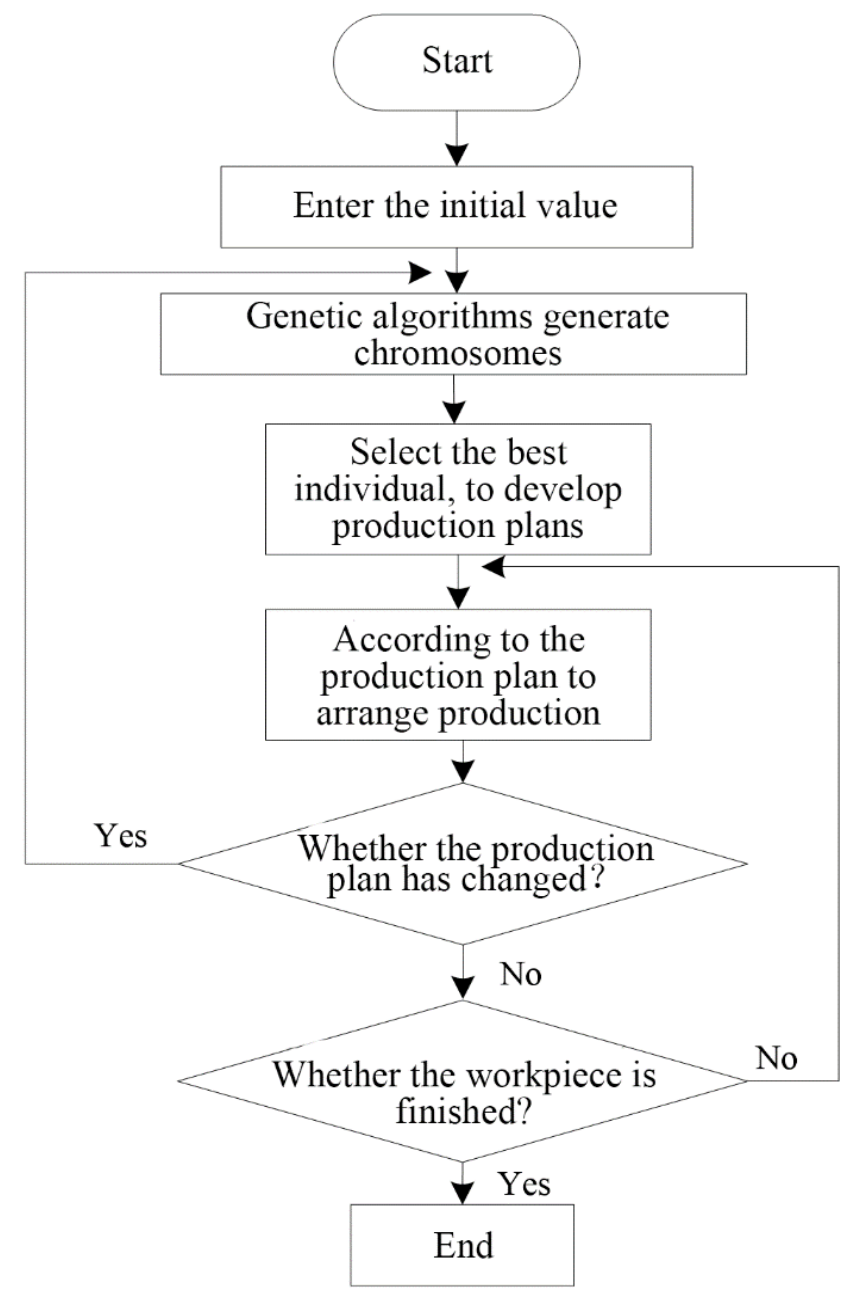

Figure 4: Flow chart of the algorithm.

If there is a delay in manufacture of workpieces in the workshop, the chromosome should be regenerated according to the genetic algorithm and based on the scheduling rules, and the production plan should be readjusted. Insert urgent workpiece 6 when $t=16$; machine 1 is damaged when $t=9$ and repaired when $t=16$. The completion time of workpieces is shown in Table IV.

Table IV: Dynamic scheduling instance under dynamic circumstance.

\begin{tabular}{|c|c|c|c|}
\hline Workpiece number & Delivery time & Complete time & Workpiece completed in advance \\
\hline $\mathbf{1}$ & 29 & 30 & -1 \\
\hline $\mathbf{2}$ & 30 & 23 & 7 \\
\hline $\mathbf{3}$ & 33 & 35 & -2 \\
\hline $\mathbf{4}$ & 34 & 36 & -2 \\
\hline $\mathbf{5}$ & 35 & 39 & -4 \\
\hline $\mathbf{6}$ & 40 & 42 & -2 \\
\hline
\end{tabular}


It can be seen from the table that under the guidance of genetic algorithm, the dynamic scheduling of production workshop has the ability to complete production ahead of schedule in the dynamic production environment. In the simulation research, the overall utilization rate of the machine is high, only the workpiece 2 is delayed for the production, and the overall delay time is small, which meets the requirement for actual production of the workshop.

\section{CONCLUSIONS}

In order to improve the market competitiveness and the workpiece production efficiency of manufacturing enterprises, it has become an inevitable trend to simulate the production of the workshop and realize the scientific guidance to the production of the workshop. In this study, the production workshop scheduling is taken as the research object; the simulation research of the production workshop scheduling is realized by effectively combining the scheduling rules and genetic algorithm. The main contents and prospects of this study are as follows:

(1): The principle and rules of workshop scheduling are expounded, and the dynamic algorithm model of production workshop scheduling is established.

(2): Based on the idea of genetic algorithm, the scheduling rules are taken as the genetic factors to realize the simulation research of production workshop scheduling. Simulation results show the stability and effectiveness of the genetic algorithm.

(3): The man-machine interaction of simulation system is realized by VC computer technology and Matlab tool.

(4): Facing the changeable environment and resource constraints of production workshop, the simulation research of production workshop scheduling should be further promoted in order to improve the scientific guidance of production.

\section{ACKNOWLEDGEMENTS}

This research has been supported in part by the Jiangxi Province Electronic Commerce high level Engineering Research Center, China. Additionally, supported by Yiwu Industrial and Commercial College, and also supported by Research on Adoption and Sustained Use of Mobile Social Network Users (71261008, National Natural Science Foundation of China), Research on the Influence of Mobile Social Network on Network Public Opinion and Its Governance (14CTQ031, National Social Science Foundation of China), Exploring the Construction of Entrepreneurship College Based on Mixed Ownership Model (taking Yiwu Institute of Business and Technology as an example) (jg20160349, Zhejiang Province Higher Education Teaching Reform Project), and An Empirical Study on the Mining and Sharing of Tacit Knowledge in Higher Vocational Education (Y201432371, Zhejiang Provincial Department of Education). The authors are indebted to Yong Guo and Juling Ding for their assistance on earlier drafts, and we also would like to thank many colleagues for their constructive criticism and helpful suggestions for improving the overall quality of this paper.

\section{REFERENCES}

[1] Gattiker, T. F. (2007). Enterprise resource planning (ERP) systems and the manufacturingmarketing interface: an information-processing theory view, International Journal of Production Research, Vol. 45, No. 13, 2895-2917, doi:10.1080/00207540600690511

[2] Tang, M.; Gong, D.; Liu, S.; Zhang, H. (2016). Applying multi-phase particle swarm optimization to solve bulk cargo port scheduling problem, Advances in Production Engineering \& Management, Vol. 11, No. 4, 299-310, doi:10.14743/apem2016.4.228

[3] Fabian, B.; Kunz, S.; Konnegen, M.; Müller, S.; Günther, O. (2012). Access control for semantic data federations in industrial product-lifecycle management, Computers in Industry, Vol. 63, No. 9, 930-940, doi:10.1016/j.compind.2012.08.015 
[4] Florescu, A.; Bratcu, A. I.; Munteanu, I.; Bacha, S. (2012). Energy management system within electric vehicles using ultracapacitors: an LQG-optimal-control-based solution, IFAC Proceedings Volumes, Vol. 45, No. 25, 229-234, doi:10.3182/20120913-4-it-4027.00033

[5] Pan, Q.-K.; Tasgetiren, M. F.; Suganthan, P. N.; Chua, T. J. (2011). A discrete artificial bee colony algorithm for the lot-streaming flow shop scheduling problem, Information Sciences, Vol. 181, No. 12, 2455-2468, doi:10.1016/j.ins.2009.12.025

[6] Pacciarelli, D.; Pranzo, M. (2004). Production scheduling in a steelmaking-continuous casting plant, Computers \& Chemical Engineering, Vol. 28, No. 12, 2823-2835, doi:10.1016/ j.compchemeng.2004.08.031

[7] Janak, S. L.; Floudas, C. A.; Kallrath, J.; Vormbrock, N. (2006). Production scheduling of a large-scale industrial batch plant. I. Short-term and medium-term scheduling, Industrial \& Engineering Chemistry Research, Vol. 45, No. 25, 8234-8252, doi:10.1021/ie0600588

[8] Janak, S. L.; Floudas, C. A.; Kallrath, J.; Vormbrock, N. (2006). Production scheduling of a large-scale industrial batch plant. II. Reactive scheduling, Industrial \& Engineering Chemistry Research, Vol. 45, No. 25, 8253-8269, doi:10.1021/ie0600590

[9] Harjunkoski, I.; Maravelias, C. T.; Bongers, P.; Castro, P. M.; Engell, S.; Grossmann, I. E.; Hooker, J.; Mendez, C.; Sand, G.; Wassick, J. (2014). Scope for industrial applications of production scheduling models and solution methods, Computers \& Chemical Engineering, Vol. 62, 161-193, doi:10.1016/j.compchemeng.2013.12.001

[10] Huang, X. R.; Dai, W.; Du, B. G. (2016). Resource-constrained project scheduling problem for large complex equipment: a hybrid approach using Pareto genetic algorithm and interval-valued intuitionistic fuzzy sets, Academic Journal of Manufacturing Engineering, Vol. 14, No. 1, 12-21

[11] Maulik, U.; Bandyopadhyay, S. (2000). Genetic algorithm-based clustering technique, Pattern Recognition, Vol. 33, No. 9, 1455-1465, doi:10.1016/S0031-3203(99)00137-5

[12] Wen, F.; Xiao, J.; Huang, C.; Xia, X. (2018). Interaction between oil and US dollar exchange rate: nonlinear causality, time-varying influence and structural breaks in volatility, Applied Economics, Vol. 50, No. 3, 319-334, doi: $10.1080 / 00036846.2017 .1321838$

[13] Hartmann, S. (1998). A competitive genetic algorithm for resource-constrained project scheduling, Naval Research Logistics, Vol. 45, No. 7, 733-750, doi:10.1002/(sici)15206750(199810)45:7<733::aid-nav5>3.0.co;2-c

[14] Wang, R.-Z.; Lin, C.-F.; Lin, J.-C. (2001). Image hiding by optimal LSB substitution and genetic algorithm, Pattern Recognition, Vol. 34, No. 3, 671-683, doi:10.1016/s0031-3203(00)00015-7

[15] Jia, Z. Y.; Lu, X. H.; Yang, J. Y.; Jia, D. F. (2011). Research on job-shop scheduling problem based on genetic algorithm, International Journal of Production Research, Vol. 49, No. 12, 3585-3604, doi:10.1080/00207543.2010.481293

[16] Barbulescu, L.; Howe, A. E.; Whitley, L. D.; Roberts, M. (2006). Understanding algorithm performance on an oversubscribed scheduling application, Journal of Artificial Intelligence Research, Vol. 27, 577-615, doi:10.1613/jair.2038

[17] Behnam, F.; Lee, L.; Romeo, M. (2012). Genetic algorithm optimisation of an integrated aggregate production-distribution plan in supply chains, International Journal of Production Research, Vol. 50, No. 1, 81-96, doi:10.1080/00207543.2011.571447

[18] Tang, M.; Gong, D.; Liu, S.; Lu, X. (2017). Finding key factors affecting the locations of electric vehicle charging station location: a simulation and ANOVA approach, International Journal of Simulation Modelling, Vol. 16, No. 3, 541-554, doi:10.2507/IJSIMM16(3)CO15

[19] Wang, Y.; Lu, Y. J.; Si, C. D.; Sung, P. (2016). Tire-pavement coupling dynamic simulation under tire high-speed-rolling condition, International Journal of Simulation Modelling, Vol. 15, No. 2, 236-248, doi:10.2507/IJSIMM15(2)4.332 